\title{
Pädiatrische Echokardiografie
}

\section{Eine Rezension von Univ.-Prof. Dr. Andreas Gamillscheg}

Die Echokardiographie stellt heutzutage die wichtigste Methode in der Diagnostik und Verlaufsbeurteilung von angeborenen Herzfehlern vom Fetus bis zum Erwachsenenalter sowie von erworbenen Herzerkrankungen im Kindesalter dar. Das von drei renommierten deutschen Kinderkardiologen im Thiemeverlag herausgegebene Buch „Pädiatrische Echokardiografie" ist eines der ersten deutschsprachigen Bücher, das sich ausschließlich und umfangreich dieser komplexen Materie widmet.

\section{Grundlagen}

In didaktisch hervorragender Weise werden im ersten Abschnitt die Leser ausführlich in die Grundzüge der pädiatrischen Echokardiografie mit den technischen und anatomischen Grundlagen, den verschiedenen Standardschnitten, den morphometrischen Analysen und den hämodynamischen Messungen mit dem jeweils klinisch praktischen Bezug eingeführt. Hervorragende Schemata mit korrespondierenden echokardiografischen Bildern erleichtern den Zugang und überwinden die ersten Schritte auch für noch unerfahrene Untersucher. Altersabhängige Normalwerte in übersichtlichen Tabellen und Graphiken ergänzen diesen Abschnitt.

\section{Einfache und komplexe Herzfehler}

Im zweiten Teil des Buches wird auf sämtliche einfache und komplexe Herzfehler sehr systematisch und leicht verständlich eingegangen. Es wird jeweils der einzelne Herzfehler zuerst kurz in Form seiner pathologischen Anatomie, Hämodynamik, mit dem klinischen Verlauf und schließlich mit seiner Therapie vorgestellt und anschließend ausführlich auf die echokardiografische Evaluierung eingegangen. Graphisch hochwertige Schemata der einzelnen
Herzfehler mit den entsprechenden typischen echokardiografischen Bildern ergänzen den Text für die Leser sehr anschaulich. Besonders für die alltägliche Praxis hervorzuheben sind die bei jedem Herzfehler jeweils noch gesondert angeführten Besonderheiten bei Erwachsenen sowie spezielle echokardiografische Fragestellungen vor und nach einer Herzkatheterintervention oder Operation.

Im Text eingefügte markierte Checklisten und Hinweise fassen die wichtigsten Punkte jedes vorgestellten Herzfehlers übersichtlich noch einmal zusammen. Auch auf etwaige Fallstricke wird jeweils hingewiesen. In eigenen Kapiteln werden die echokardiografische Beurteilung der pulmonalarteriellen Hypertonie und des Fontankreislaufs, die Cardiomyopathien sowie schließlich die erworbenen Herzerkrankungen im Kindesalter behandelt. Standardschnitte der transösophagealen Echokardiografie werden in einem eigenen Kapitel ebenfalls kurz vorgestellt. Besonders hervor zu streichen sind schließlich spezielle Kapitel, die gezielt die Primär- und Notfallsdiagnostik in der Neonatologie und der pädiatrischen Intensivmedizin sehr praxisnah und prägnant vorstellen. Jedes Buchkapitel wird mit Hinweisen auf die aktuellste bzw. wichtigste weiterführende Literatur abgeschlossen.

\section{Für die Praxis geschrieben}

Das Buch ist für die Praxis geschrieben, ohne den notwendigen theoretischen Hintergrund zu vernachlässigen. Insbesondere ist den Autoren der Zusammenhang zwischen dem jeweiligen echokardiographischen Befund und der Hämodynamik bzw. der daraus folgenden klinischen Konsequenz außerordentlich gut gelungen. Durch den strukturierten und didaktisch ausge- zeichneten Aufbau und das ausführliche und anschauliche Bildmaterial (ergänzt durch die Möglichkeit entsprechende Videos auch über eine Online Plattform zu studieren) eignet sich das Buch sehr gut zum Erlernen dieser zum Teil komplexen Materie. Aber auch erfahrene KinderkardiologInnen werden gerne zur raschen Orientierung auf dieses Buch zurückgreifen. Durch die Praxisnähe wird das Buch auch bei KollegInnen aus der Kinderherzchirurgie, der Erwachsenenkardiologie, der Neonatologie und der Pädiatrischen Intensivmedizin auf Interesse stoßen. Dieses Buch wird sich als deutschsprachiges Standardwerk in der pädiatrischen Echokardiografie etablieren, weshalb es allen Kolleglnnen, die mit herzkranken Kindern oder angeborenen Herzfehlern befasst sind, uneingeschränkt und dringend zu empfehlen ist.

\section{Korrespondenzadresse}

Univ.-Prof. Dr. Andreas Gamillscheg Univ.-Klinik für Kinder- und Jugendheilkunde, Klin. Abteilung für Pädiatrische Kardiologie, Medizinische Universität Graz Auenbruggerplatz 34/2,

8036 Graz, Österreich

andreas.gamillscheg@medunigraz.at

Hinweis des Verlags. Der Verlag bleibt in Hinblick auf geografische Zuordnungen und Gebietsbezeichnungen in veröffentlichten Karten und Institutsadressen neutral.

Paediatr. Paedolog. 2020 · 55:223 https://doi.org/10.1007/s00608-02000825-x

(c) Springer-Verlag GmbH Austria, ein Teil von Springer Nature 2020

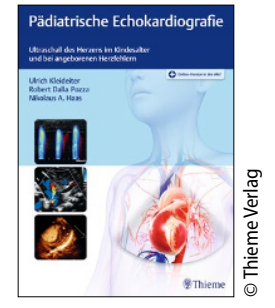

